\title{
Optimized balance rehabilitation training strategy for the elderly through an evaluation of balance characteristics in response to dynamic motions
}

This article was published in the following Dove Press journal:

Clinical Interventions in Aging

14 October 2015

Number of times this article has been viewed

\author{
HoHyun Jung' \\ Keyoung Jin Chun ${ }^{2}$ \\ Jaesoo Hong ${ }^{2}$ \\ Dohyung Lim' \\ 'Department of Mechanical \\ Engineering, Sejong University, Seoul, \\ Republic of Korea; ${ }^{2}$ Smart Welfare \\ Technology Research Group, Korea \\ Institute of Industrial Technology, \\ Cheonan, Republic of Korea
}

\begin{abstract}
Balance is important in daily activities and essential for maintaining an independent lifestyle in the elderly. Recent studies have shown that balance rehabilitation training can improve the balance ability of the elderly, and diverse balance rehabilitation training equipment has been developed. However, there has been little research into optimized strategies for balance rehabilitation training. To provide an optimized strategy, we analyzed the balance characteristics of participants in response to the rotation of a base plate on multiple axes. Seven male adults with no musculoskeletal or nervous system-related diseases (age: $25.5 \pm 1.7$ years; height: $173.9 \pm 6.4 \mathrm{~cm}$; body mass: $71.3 \pm 6.5 \mathrm{~kg}$; body mass index: $23.6 \pm 2.4 \mathrm{~kg} / \mathrm{m}^{2}$ ) were selected to investigate the balance rehabilitation training using customized rehabilitation equipment. Rotation of the base plate of the equipment was controlled to induce dynamic rotation of participants in the anterior-posterior, right-diagonal, medial-lateral, and left-diagonal directions. We used a three-dimensional motion capture system employing infrared cameras and the Pedar Flexible Insoles System to characterize the major lower-extremity joint angles, center of body mass, and center of pressure. We found statistically significant differences between the changes in joint angles in the lower extremities in response to dynamic rotation of the participants $(P<0.05)$. The maximum was greater with anterior-posterior and medial-lateral dynamic rotation than with that in other directions $(P<0.05)$. However, there were no statistically significant differences in the frequency of center of body mass deviations from the base of support $(P>0.05)$. These results indicate that optimizing rotation control of the base plate of balance rehabilitation training equipment to induce anterior-posterior and medial-lateral dynamic rotation preferentially can lead to effective balance training. Additional tests with varied speeds and ranges of angles of base plate rotation are expected to be useful as well as an analysis of the balance characteristics considering a balance index that reflects the muscle activity and cooperative characteristics.
\end{abstract}

Keywords: optimized strategy, balance rehabilitation training, balance characteristics, maximum deviation, deviation frequency

\section{Introduction}

Balance is important in daily activities and is known to be a requirement for maintaining an independent lifestyle in the elderly. ${ }^{1}$ However, as aging progresses, the sensory and motor systems that are involved in the stability of postures and control of body directions decline, as does the strength of the major muscles required for maintaining balance, ultimately leading to a reduction in balance ability. ${ }^{2}$ During the aging process, particularly from 30 to 70 years, maximum muscle strength declines by around $30 \%-50 \%,{ }^{3}$ and the lower-extremity muscle strength declines by around $1 \%-4 \%$ each year from the age of 50 years. ${ }^{4}$ When balance is disrupted in the elderly, the decline in muscle strength makes it difficult to generate sufficient joint moment to achieve
Correspondence: Dohyung Lim

Department of Mechanical Engineering, Sejong University, 209 Neungdong-ro,

Gwangjin-gu, Seoul I43-747,

Republic of Korea

Tel +82 234083672

Fax +82 234084333

Email dli349@sejong.ac.kr 
balance recovery. ${ }^{5,6}$ In addition, a decline in balance ability is reported to decrease physical function and to increase the likelihood of falling. ${ }^{7}$ In the US, 2.2 million elderly patients received treatment for fall injuries in 2009, 19,000 of whom were reported to have died. ${ }^{89}$ The relevant medical costs were reported to be 23.3 billion dollars in 2008, and it is predicted that this will increase to 55 billion dollars worldwide in $2020 .^{8}$ It follows that falls are a socially important issue, ${ }^{10}$ and research into methods of preventing falls by improving muscle strength and balance ability is thereby warranted.

Various methods of analyzing balance characteristics have been reported as well as studies into balance rehabilitation training using a moving base plate. ${ }^{11-16}$ Ogaya et al ${ }^{11}$ and Martínez-Amat et $\mathrm{al}^{12}$ used balance training equipment with an unstable base plate to test the efficiency of balance rehabilitation training by evaluating the balance ability and the level of improvement in physical functions of elderly subjects. Maeda et $\mathrm{al}^{13}$ reported balance rehabilitation training equipment whereby anterior-posterior (AP) and medial-lateral (ML) dynamic rotation could be induced by randomly controlling the order of the motion of the base plate, which could rotate on the transverse and sagittal axes. Tsai et $\mathrm{al}^{14}$ reported the use of balance rehabilitation training equipment that could create sudden perturbations, inducing the AP dynamic rotation via constant sequential control of a base plate that could rotate on the transverse and sagittal axes. The authors of these two studies ${ }^{13,14}$ demonstrated the potential of such training equipment to improve balance ability; however, balance training that involves an unstable base plate has the potential to provide random rotation according to the intent of the user. Because of this, there are limitations in terms of the control of rotation of the base plate required to maximize the improvement in balance ability. Furthermore, when the base plate is rotated in a specific axis, only AP or ML dynamic rotation was considered in the investigations of balance ability and muscle strength. Hence, there is scope to optimize the rotation control of the base plate to maximize the improvements in balance ability. Cadore et a ${ }^{15}$ and Halvarsson et $\mathrm{al}^{16}$ reported that optimized balance training programs in which the intensity, frequency, and direction are organized strategically are expected to provide efficient improvements of balance ability.

As a pilot study to provide an optimized strategy for control of the base plate of balance rehabilitation training equipment, we analyzed balance characteristics in response to changes in the rotation of the base plate and derived a basic strategy for optimizing the possibility of improvement of balance ability in the elderly. It was conducted based on the hypothesis that human balance characteristics generally respond differently to the rotation of the base plate, resulting in an optimized combination of rotations, which may generate a maximum effect of balance rehabilitation training.

\section{Materials and methods Participants}

Seven male adults aged $25.5 \pm 1.7$ years, with an average height of $173.9 \pm 6.4 \mathrm{~cm}$, average body mass of $71.3 \pm 6.5 \mathrm{~kg}$, and average body mass index of $23.6 \pm 2.4 \mathrm{~kg} / \mathrm{m}^{2}$, and with no musculoskeletal or nervous system-related diseases were selected for the study. The risks of the tests were fully explained to the participants and the tests were conducted after receiving signed consent forms. The experimental procedures and the selection of the participants were approved by the Sejong University Institutional Review Board (IRB No SJU-2015-002).

\section{Experiment configuration and procedure}

Figure 1 shows the balance rehabilitation training equipment, which was customized to induce dynamic rotation of participants. This equipment was designed to allow controlled rotation of the base plate within a range of $\pm 15^{\circ}$ via six links that were connected to the base plate area, which could move $\pm 0.1 \mathrm{~m}$ in the vertical direction. The equipment was customized so that rotation of the base plate could be controlled in random directions to identify optimal strategies for balance rehabilitation training through analysis of balance characteristics related to the dynamic rotation of the participants, representing motions that commonly occur during various daily activities. The base plate was rotated on the transverse, right-diagonal (RD), sagittal, and left-diagonal (LD) axes to induce AP, RD, ML, and LD dynamic rotation, respectively, as shown in Figure 2. The maximum range of the rotation was $\pm 9^{\circ}$ (for safety) and the participants were allowed to perform dynamic rotation within their ability to maintain their balance. The tests were repeated five times for each movement, and three times per participant to minimize measurement errors. To minimize the effects of fatigue, the participants were given 10-minute breaks between tests.

\section{Analyses of dynamic rotation and balance characteristics}

To quantify the dynamic rotation of the participants depending on the rotation of the base plate, we used a threedimensional (3D) motion capture system with eight infrared cameras (T-10s; VICON Motion System Ltd., Oxford, UK; sampling rate: $100 \mathrm{~Hz}$ ), as shown in Figure 3. Based on the 


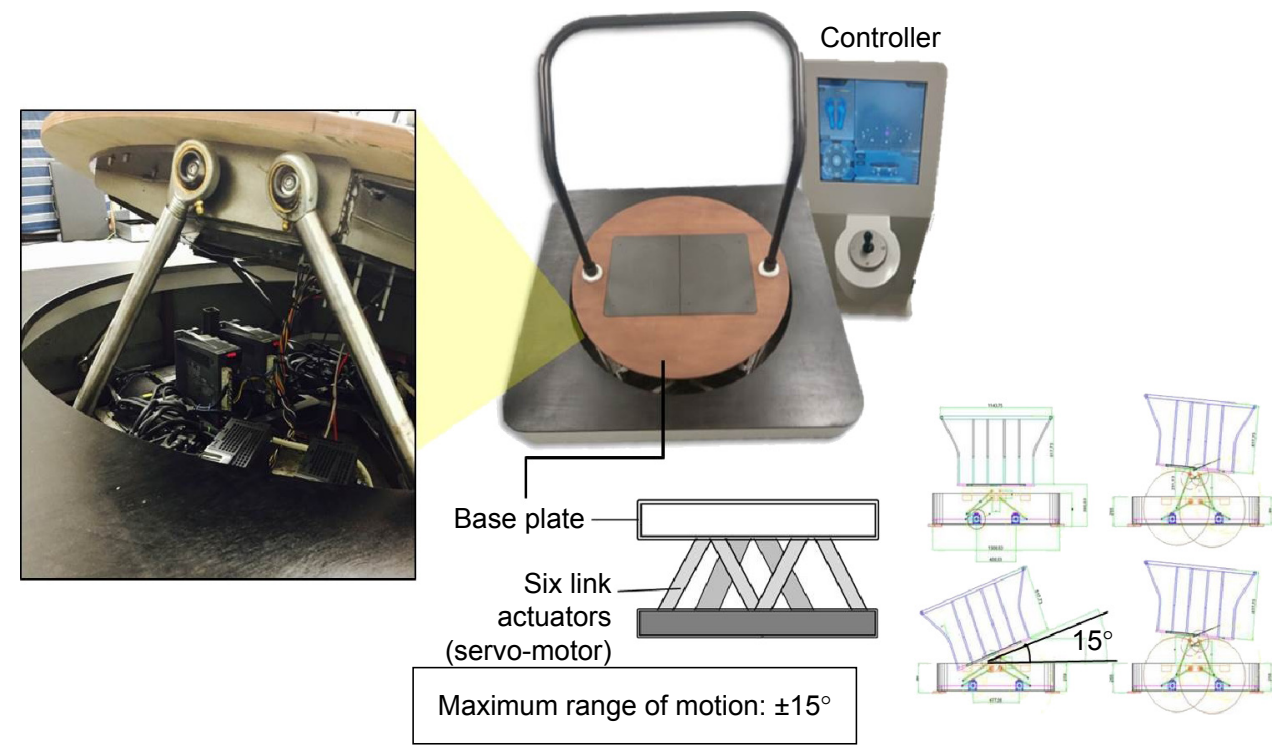

Figure I Photographs and schematic diagrams of the customized balance rehabilitation training equipment.

information describing the dynamic rotation of participants obtained using the 3D motion capture system, changes in the trajectory and velocity of the center of body mass (COM) as well as changes in the major lower-extremity joint angles (ie, the ankle joint, knee joint, and hip joint) were analyzed and evaluated using Nexus software (VICON Motion System Ltd.).
The Pedar Flexible Insoles System (Novel GmbH, Munich, Germany; sampling rate: $100 \mathrm{~Hz}$ ) was used to measure the center of pressure (COP), and the base of support (BOS) was established. To analyze balance characteristics, changes in the trajectory of the COM were projected onto the BOS to determine the balance indices of the participant. Balance indices were defined using the maximum deviation,

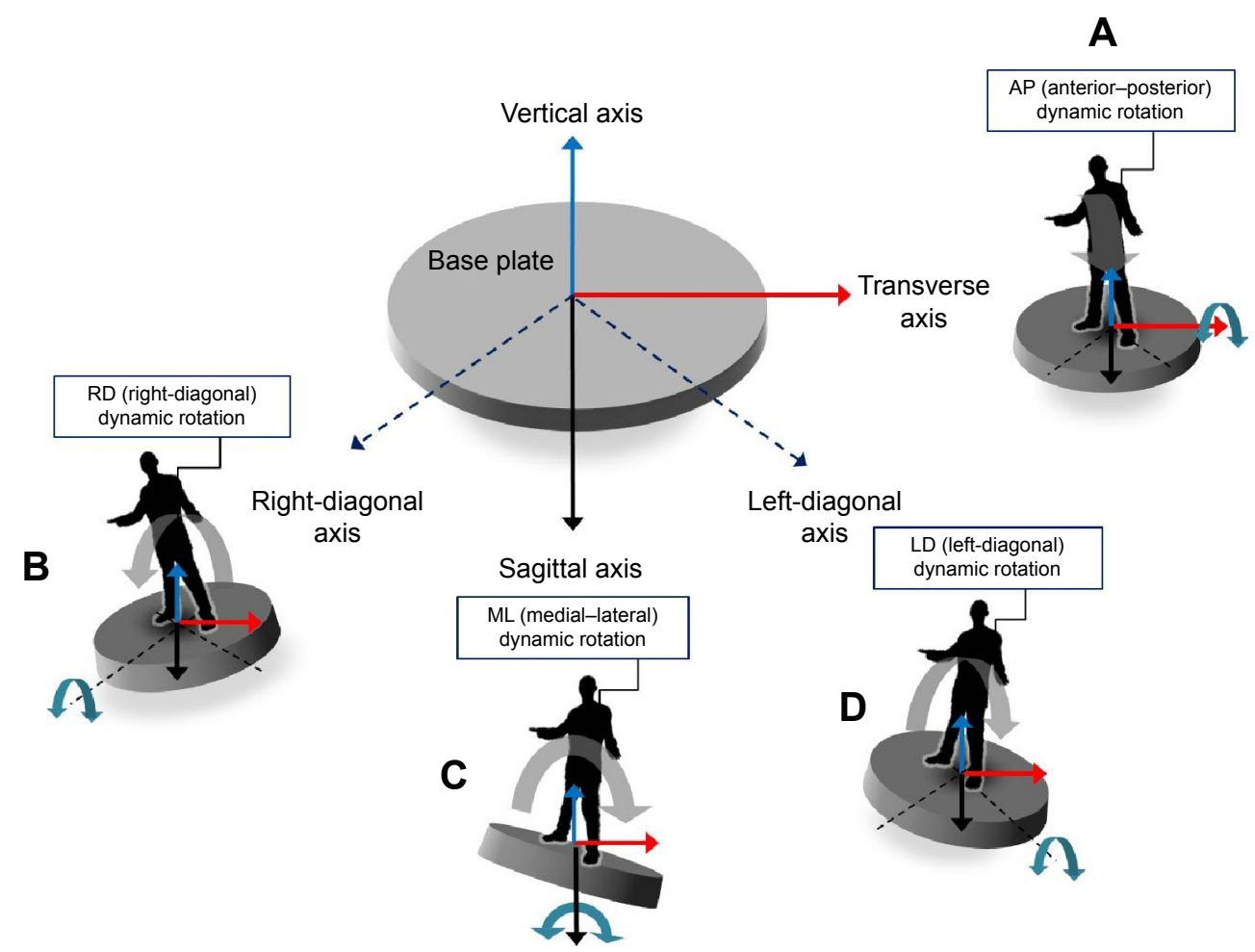

Figure 2 The dynamic rotation of participants induced by rotation of the base plate: (A) AP, (B) RD, (C) ML, and (D) LD. 


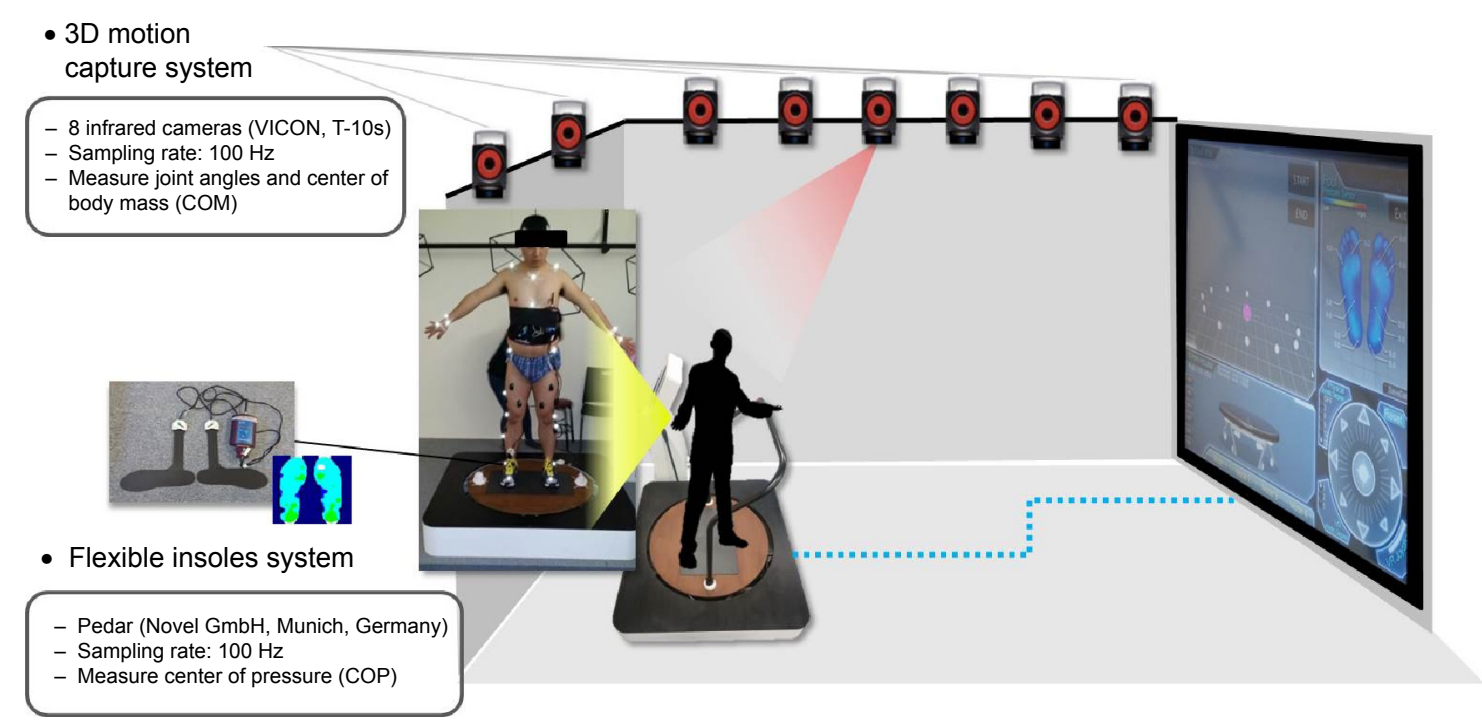

Figure 3 The experimental configuration used to generate and characterize the motion of the participants.

which describes the maximum displacement of the projected COM relative to the BOS, as well as the deviation frequency, which describes the frequency of deviation of the projected COM from the BOS in these deviations.

\section{Statistical analysis}

To characterize the significance of differences in changes in major joint angles and balance characteristics depending on the dynamic rotation characteristics of the participants, one-way analysis of variance (ANOVA) was performed with Scheffe's post hoc analysis using the SPSS software package (IBM Corporation, Armonk, NY, USA). Statistical significance was considered as $P<0.05$.

\section{Results}

\section{Changes in the major lower-extremity joint angles in response to dynamic rotation}

Figure 4 shows the changes in the major lower-extremity joint angles depending on the dynamic rotation of the participants. Where dynamic rotation was induced by rotation of the base plate, we confirmed that the changes that were induced in the lower-extremity joint angles overall differed significantly with the type of dynamic rotation $(P<0.05)$. With AP dynamic rotation, relatively large changes in dorsiflexion-plantar flexion occurred in the ankle joint $\left(22.7^{\circ} \pm 5.2^{\circ}\right)$; with ML dynamic rotation, relatively large changes in external-internal rotation occurred in the hip joint $\left(22.3^{\circ} \pm 6.6^{\circ}\right)(P<0.05)$. With $\mathrm{LD}$ and $\mathrm{RD}$ dynamic rotation, relatively large changes occurred in flexion-extension of the knee joint (LD dynamic rotation: $18.4^{\circ} \pm 6.4^{\circ}$; RD dynamic rotation: $\left.20.7^{\circ} \pm 8.1^{\circ}\right)(P<0.05)$.

\section{Balance characteristics in response to dynamic rotation}

Figure 5 shows the results of the balance characteristics of the participants depending on the rotation of the base plate. The maximum deviations with AP and ML dynamic rotation were larger than those with $\mathrm{RD}$ and $\mathrm{LD}$ dynamic rotation (AP dynamic rotation: $60.4 \% \pm 15.6 \%$; RD dynamic rotation: $35.6 \% \pm 9.9 \%$; ML dynamic rotation: $58.6 \% \pm 13.5 \%$; LD dynamic rotation: $14.9 \% \pm 6.0 \%)(P<0.05)$. However, the deviation frequency was similar for all directions; that is, $27.7 \% \pm 8.5 \%$ for AP dynamic rotation, $28.6 \% \pm 20.3 \%$ for $\mathrm{RD}$ dynamic rotation, $23.0 \% \pm 10.9 \%$ for ML dynamic rotation, and $31.4 \% \pm 20.8 \%$ for LD dynamic rotation, and there was no significant difference among the types of dynamic rotation $(P>0.05)$. Therefore, although there was no difference in the deviation frequency of the COM from the BOS for each type of dynamic rotation, larger deviations were induced with $\mathrm{AP}$ and ML dynamic rotation. In addition, the velocities of COMs did not differ significantly with the type of dynamic rotation (AP dynamic rotation: $3.0 \pm 1.6 \mathrm{~cm} / \mathrm{s}$; RD dynamic rotation: $3.2 \pm 0.7 \mathrm{~cm} / \mathrm{s}$; ML dynamic rotation: $3.5 \pm 0.8 \mathrm{~cm} / \mathrm{s}$; LD dynamic rotation: $3.9 \pm 0.9 \mathrm{~cm} / \mathrm{s})(P>0.05)$.

\section{Discussion}

Balance indices based on changes in the COM or COP have been used to analyze balance characteristics from a biomechanical perspective. ${ }^{11,17,18}$ However, to provide a 

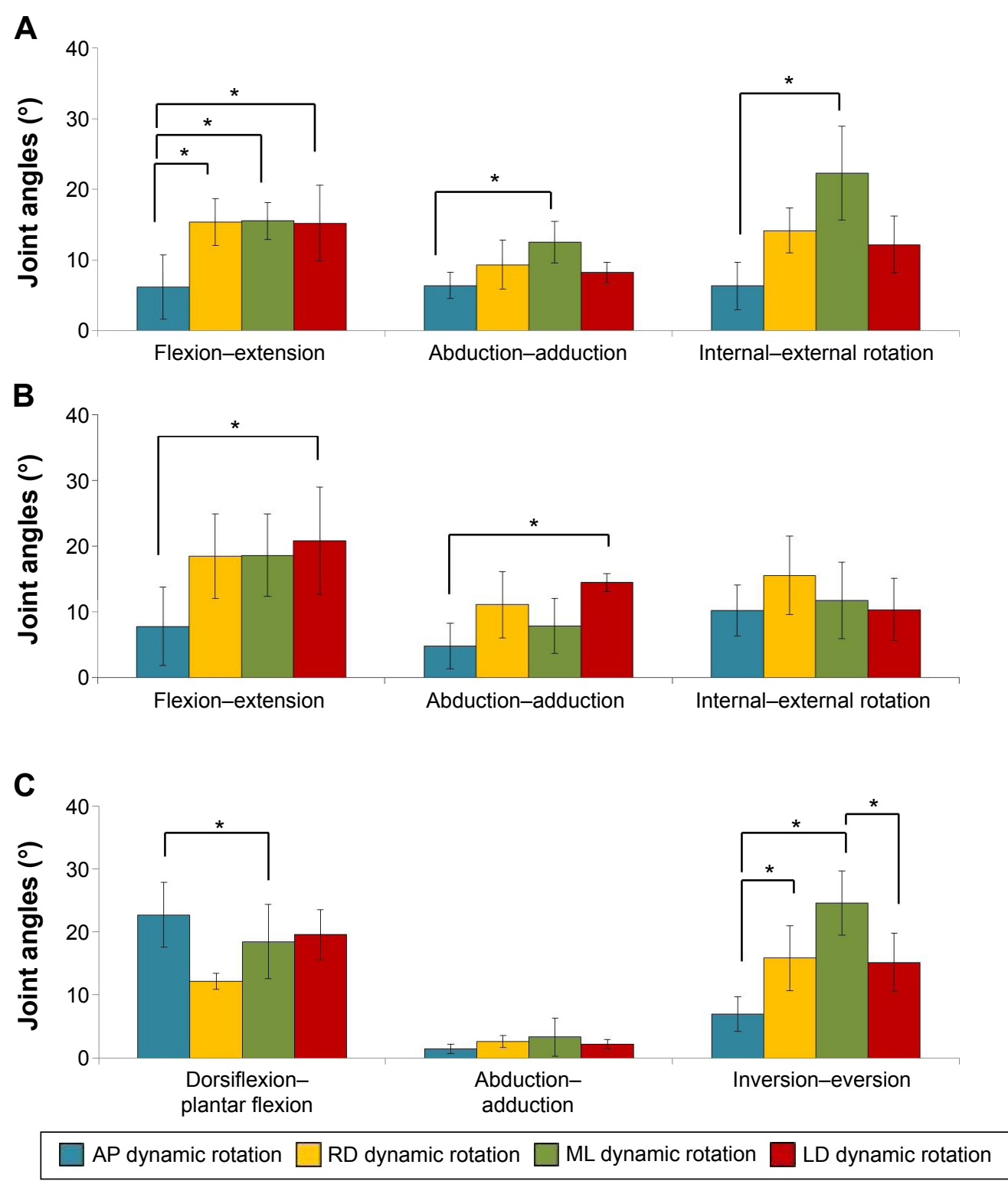

Figure 4 Variations in the joint angles of the lower extremities in response to rotation of the base plate of the balance rehabilitation training equipment. Notes: The hip joint (A), the knee joint (B), and the ankle joint $(\mathbf{C})$. *Statistical significance $(P<0.05)$.

Abbreviations: AP, anterior-posterior; RD, right-diagonal; ML, medial-lateral; LD, left-diagonal.

more accurate analysis of balance characteristics, various balance indices may be combined. Hur et a $1^{19}$ proposed a balance index that combined the COM and COP, and Clark et $\mathrm{a}^{17}$ reported and evaluated a balance index that incorporated dynamic weight bearing asymmetry assessment (for both feet) and the trajectory velocity of the COP. Hof et $\mathrm{al}^{20}$ reported a relation between the BOS and the COM projected onto the BOS and used a balance index to analyze balance characteristics during standing.

Here, we established two balance indices that were considered the most suitable for our test environment and based on that used by Hof et al. ${ }^{20}$ In general, maintaining balance is defined as maintaining the projected trajectory of the COM within the BOS. Hence, a balance index considering a combination of BOS and COM is likely to provide reliable information for an analysis of balance characteristics. The BOS is an important factor in the maintenance of postures while standing and has been reported to be correlated with posture maintenance. ${ }^{21}$ Furthermore, the COM has been reported to be directly associated with the characteristic motion of the upper body, which is involved in maintaining balance when perturbations occur in response to an unstable base plate. ${ }^{18}$ We established a balance index that reflects both the trajectory of the COM and the size of the BOS to obtain 

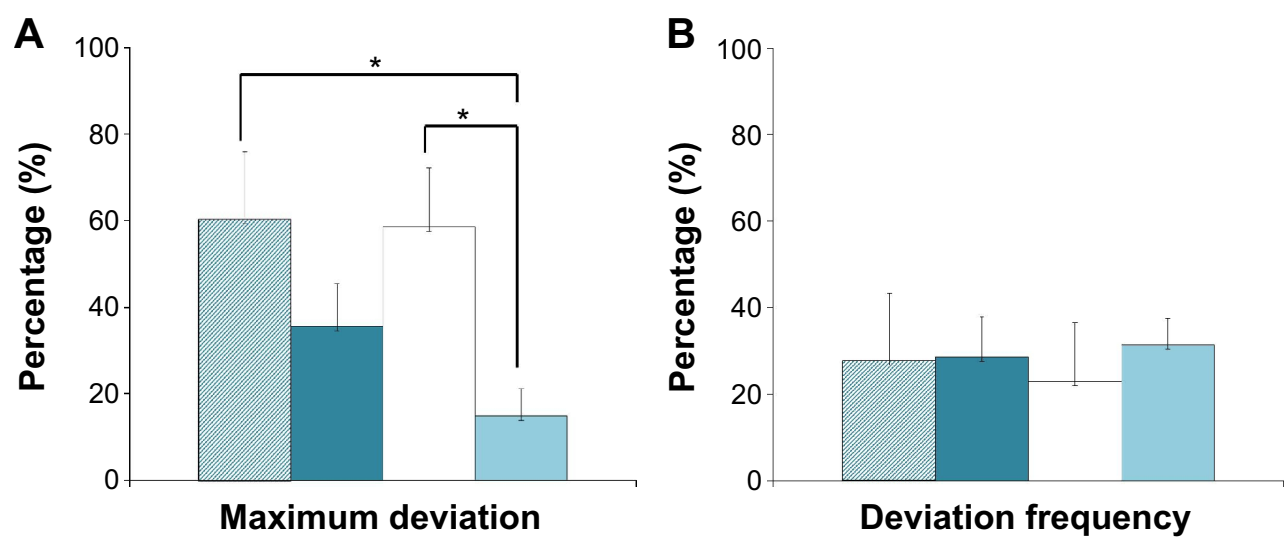

$\square$ AP dynamic rotation $\square$ RD dynamic rotation $\sqsubset$ ML dynamic rotation $\square$ LD dynamic rotation

Figure 5 Results of balance characteristics in response to rotation of the base plate.

Notes: The maximum deviation $(\mathbf{A})$ and the deviation frequency $(\mathbf{B})$. *Statistical significance $(P<0.05)$

Abbreviations: AP, anterior-posterior; RD, right-diagonal; $M L$, medial-lateral; $L D$, left-diagonal.

an accurate assessment of balance characteristics during dynamic rotation.

Depending on the balance characteristics, the COM trajectory deviated from the BOS with maximum displacement in response to AP and ML dynamic rotation. Particularly with AP dynamic rotation, the range of the ankle joint angles (which is involved in maintaining the balance of the human body $)^{22}$ was shown to be larger for extension and flexion compared with other joints. Maeda et $\mathrm{al}^{13}$ reported that the range of ankle joint angles was larger in the rotation of a base plate on the transverse axis than the sagittal axis, which is consistent with the results of this work. Furthermore, with AP dynamic rotation, the ankle joint is critical in maintaining a standing posture, providing stability to the body. ${ }^{23}$ Thus, AP dynamic rotation is expected to increase the range of joint angles of the ankle as well as improve the strength of related muscles. These results suggest that triggering such a rotation of the base plate on the transverse axis induces greater AP dynamic rotation of the user and ultimately promotes more efficient balance training.

With ML dynamic rotation, the COM deviated significantly from the BOS, and the range of hip joint angles was larger for both internal and external rotation than for other joints. Horak et $\mathrm{al}^{24}$ reported that the hip joint is important in maintaining balance in the elderly. Therefore, inducing ML dynamic rotation during balance training in the elderly may increase the range of motion of the hip joint. This is likely to be effective in improving balance ability in the elderly. However, because the feet are placed in parallel, it has been reported that the size of the user's BOS appears to be smaller in ML dynamic rotation than in AP dynamic rotation, and thus maintaining balance is more difficult in ML dynamic rotation. ${ }^{21}$ For these reasons, if motion that can increase the size of the user's BOS is supplemented during balance training (including ML dynamic rotation) we may expect efficient improvements in balance ability.

As the extent of deviation of the COM from the BOS increases, the likelihood of falling increases. In situations where balance is lost, a feedback mechanism that allows the COM to return to the BOS is rapidly activated by the nervous and musculoskeletal systems to recover balance. ${ }^{23}$ It follows that repeatedly providing the allowable maximum rotation to the user without the risk of falling is likely to stimulate the nervous and musculoskeletal systems, inducing effective balance rehabilitation training. Therefore, because AP and ML dynamic rotation generated larger deviation of the COM and larger changes in joint angles, they are more likely to induce effective balance rehabilitation compared with other types of dynamic rotation. In other words, optimized rotation control of the base plate of balance rehabilitation training equipment triggering various types of dynamic rotation (especially AP and ML dynamic rotation) is expected to be effective in improving balance ability in the elderly. However, the participants in our study were healthy young adults. If the results of this study are to be applied to balance rehabilitation training of the elderly, or those with impaired balance ability such as hemiplegic patients, it should be taken into account that these patients have a different level of response to external perturbations provided by a base plate. ${ }^{25}$ However, because of the decline in balance-maintaining function and muscle strength as aging progresses ${ }^{2}$ providing balance rehabilitation training with moderate intensity and frequency, as well 
as gradually increasing the intensity of the perturbation to improve balance, is expected to be an important aspect of effective training.

In this study, we analyzed the balance characteristics of users via various types of rotation of the base plate and described a control strategy for optimizing the improvement of balance ability. Owing to difficulties in recruitment, we used a relatively small number of participants, and the tests were performed using a base plate that rotated within a limited range of angles and at constant speed to minimize the effects of the user's balance characteristics. Furthermore, muscle activity and cooperative muscle characteristics are required to maintain balance and affect the trajectory of the COM and $\mathrm{COP},{ }^{26}$ which suggests that the level of muscle development may affect balance characteristics. It follows that both muscle activity and cooperative muscle characteristics should be considered. As part of future work, additional studies to compensate for such limitations are planned, to allow for a more accurate analysis of balance characteristics. This is expected to lead to more reliable optimization of the control strategy for the rotation of the base plate of balance rehabilitation training equipment.

\section{Conclusion}

The results of our investigation show that optimized control of the rotation of the base plate of balance rehabilitation training equipment can be used to induce AP and ML dynamic rotation to improve balance ability in the elderly. To find a better optimized strategy for controlling the rotation of the base plate, reinforced tests should be carried out in the near future to investigate factors that affect a user's balance characteristics, especially variation in the range of rotation angles of the base plate and the speed of the rotation. Furthermore, an analysis of the balance characteristics using a balance index that reflects muscle activity and cooperative muscle characteristics is warranted.

\section{Acknowledgment}

This research was supported by the Korea Institute of Industrial Technology (KITECH), Republic of Korea.

\section{Disclosure}

The authors report no conflicts of interest in this work.

\section{References}

1. Lindemann U, Muche R, Stuber M, Zijlstra W, Hauer K, Becker C. Coordination of strength exertion during the chair-rise movement in very old people. J Gerontol A Biol Sci Med Sci. 2007;62(6):636-640.
2. Kanekar N, Aruin AS. Aging and balance control in response to external perturbations: role of anticipatory and compensatory postural mechanisms. Age (Dordr). 2014;36(3):1067-1077.

3. Holviala J, Kraemer W, Sillanpää E, et al. Effects of strength, endurance and combined training on muscle strength, walking speed and dynamic balance in aging men. Eur J Appl Physiol. 2012;112(4): $1335-1347$.

4. Larsson L, Grimby G, Karlsson J. Muscle strength and speed of movement in relation to age and muscle morphology. J Appl Physiol Respir Environ Exerc Physiol. 1979;46(3):451-456.

5. Carty CP, Barrett RS, Cronin NJ, Lichtwark GA, Mills PM. Lower limb muscle weakness predicts use of a multiple- versus single-step strategy to recover from forward loss of balance in older adults. $J$ Gerontol A Biol Sci Med Sci. 2012;67(11):1246-1252.

6. Jung HH, Ko CY, Kim JS, Lee BK, Lim DH. Alterations of relative muscle contribution induced by hemiplegia: straight and turning gaits. Int J Precis Eng Manuf. 2015;16(10):2219-2227.

7. Frank JS, Patla AE. Balance and mobility challenges in older adults: implications for preserving community mobility. Am J Prev Med. 2003; 25(3 Suppl 2):157-163.

8. Ambrose AF, Paul G, Hausdorff JM. Risk factors for falls among older adults: a review of the literature. Maturitas. 2013;75(1):51-61.

9. Lim DH, Kim CY, Jung HH, Jung DY, Chun KJ. Use of the Microsoft Kinect system to characterize balance ability during balance training. Clin Interv Aging. 2015;10:1077-1083.

10. Stewart RB, Moore MT, May FE, Marks RG, Hale WE. Nocturia: a risk factor for falls in the elderly. $J$ Am Geriatr Soc. 1992;40(12): $1217-1220$.

11. Ogaya S, Ikezoe T, Soda N, Ichihashi N. Effects of balance training using wobble boards in the elderly. J Strength Cond Res. 2011;25(9): 2616-2622.

12. Martínez-Amat A, Hita-Contreras F, Lomas-Vega R, CaballeroMartínez I, Alvarez PJ, Martínez-López E. Effects of 12-week proprioception training program on postural stability, gait, and balance in older adults: a controlled clinical trial. J Strength Cond Res. 2013;27(8): 2180-2188.

13. Maeda Y, Tanaka T, Nakajima Y, Shimizu K. Analysis of postural adjustment responses to perturbation stimulus by surface tilts in the feet-together position. J Med Biol Eng. 2011;31(4):301-305.

14. Tsai YC, Hsieh LF, Yang S. Age-related changes in posture response under a continuous and unexpected perturbation. J Biomech. 2014;47(2): 482-490.

15. Cadore EL, Rodríguez-Mañas L, Sinclair A, Izquierdo M. Effects of different exercise interventions on risk of falls, gait ability, and balance in physically frail older adults: a systematic review. Rejuvenation Res. 2013;16(2):105-114.

16. Halvarsson A, Oddsson L, Olsson E, Farén E, Pettersson A, Ståhle A. Effects of new, individually adjusted, progressive balance group training for elderly people with fear of falling and tend to fall: a randomized controlled trial. Clin Rehabil. 2011;25(11):1021-1031.

17. Clark RA, McGough R, Paterson K. Reliability of an inexpensive and portable dynamic weight bearing asymmetry assessment system incorporating dual Nintendo Wii Balance Boards. Gait Posture. 2011;34(2): 288-291.

18. Horak FB. Clinical measurement of postural control in adults. Phys Ther. 1987;67(12):1881-1885.

19. Hur P, Duiser BA, Salapaka SM, Hsiao-Wecksler ET. Measuring robustness of the postural control system to a mild impulsive perturbation. IEEE Trans Neural Syst Rehabil Eng. 2010;18(4):461-467.

20. Hof A, Gazendam M, Sinke W. The condition for dynamic stability. J Biomech. 2005;38(1):1-8.

21. Amiridis IG, Hatzitaki V, Arabatzi F. Age-induced modifications of static postural control in humans. Neurosci Lett. 2003;350(3):137-140.

22. Horak FB, Nashner LM. Central programming of postural movements: adaptation to altered support-surface configurations. $J$ Neurophysiol. 1986;55(6):1369-1381. 
23. Mathiyakom $\mathrm{W}$, McNitt-Gray JL. Regulation of angular impulse during fall recovery. J Rehabil Res Dev. 2008;45(8):1237-1248.

24. Horak FB, Shupert CL, Mirka A. Components of postural dyscontrol in the elderly: a review. Neurobiol Aging. 1989;10(6):727-738.

25. Zemková E. Assessment of balance in sport: science in sport and reality. Serb J Sports Sci. 2011;5(4):127-139.
26. Laughton CA, Slavin M, Katdare K, et al. Aging, muscle activity, and balance control: physiologic changes associated with balance impairment. Gait Posture. 2003;2(18):101-108.

\section{Publish your work in this journal}

Clinical Interventions in Aging is an international, peer-reviewed journal focusing on evidence-based reports on the value or lack thereof of treatments intended to prevent or delay the onset of maladaptive correlates of aging in human beings. This journal is indexed on PubMed Central, MedLine,
CAS, Scopus and the Elsevier Bibliographic databases. The manuscript management system is completely online and includes a very quick and fair peer-review system, which is all easy to use. Visit http://www.dovepress. com/testimonials.php to read real quotes from published authors. 